\title{
APROXIMACIONES AL CONCEPTO DE MUNDO EN HUSSERL
}

\author{
APPROACHES TO THE CONCEPT OF \\ WORLD IN HUSSERL
}

\author{
Cristián Martínez-Bravo* \\ Universidad Diego Portales \\ Santiago-Chile \\ Universität zu Köln \\ Colonia-Alemania \\ Recibido diciembre de 2020/Received December, 2020 \\ Aceptado junio de 2021/Accepted June, 2021
}

\begin{abstract}
RESUMEN
El mundo es un concepto de difícil interpretación en la fenomenología de Husserl. Por un lado, dicho concepto es caracterizado como el mundo de la vida o el mundo de la cultura en su sentido más pleno (Husserl, 2008a, 288; HUA VI, 191). Este mundo puede ser pensado en el plural aunque, sin embargo, exhibe al mismo tiempo tanto un modo de ser que es de carácter horizóntico, como otro de carácter apriórico, los que, en suma, lo hacen poseer una progresión y unidad ante las cuales "el plural carece de sentido" (Husserl, 2008a, 185; HUA VI, 146). Por otro lado, el mundo también es caracterizado como siendo una adquisición subjetiva y, por consiguiente, implica un red de constitución dinámica que llega a ser lo que es a partir de la progresión desde un premundo [Vorwelt] a un mundo [Welt] (Walton, 2015; Bower, 2015). De tal manera, el mundo en Husserl aparece bajo distintas determinaciones que, incluso, pueden parecer contradictorias (Carr, 1970). En este trabajo, utilizando una diferenciación que realiza Walton (2015) entre los órdenes de la constitución y la dación, propondré que es posible reconstruir el concepto de mundo y, de este modo, aclarar su "problematicidad" (Carr, 1970). De esta manera, mostraré que tal problematicidad surge debido a una indiferenciación de los diversos estratos de la constitución del mundo, los que se articulan en investigaciones genéticas y generativas que expresan distintos niveles del análisis de dicho concepto. Concluiré que sin esta distinción que conduce a distintos niveles de análisis, se obtiene una comprensión insuficiente del concepto tal como es desarrollado por Husserl en las distintas etapas de su obra.
\end{abstract}

Palabras Clave: Mundo, mundo de la vida, horizonticidad, a priori del mundo, consistencia, progresión.

\begin{abstract}
The world is a difficult concept to interpret in Husserl's phenomenology. On the one hand, such a concept is characterized as the life-world or the world of culture in their fullest sense (Husserl, 2008a, 288; HUA VI, 191). This world can be thought in plural although, however, it exhibits at the same time, both, a horizontal manner of being and an A Priori nature, which, in sum, makes the world possess a progression and unity in front of which "the plural makes no sense" (Husserl, 2008a, 185; HUA VI, 146). On the other hand, the world is also characterized as a subjective acquisition and, for that reason, it implies a dynamic constitutional network which comes to be what it is from the progression from a pre-world [Vorwelt] to a world [Welt] (Walton, 2015; Bower, 2015). Therefore, Husserl's world appears under different determinations that may even seem contradictory (Carr, 1970). In this work, using a differentiation made by Walton (2015) between constitutional and given, I propose that it is possible to reconstruct the concept of world and, thereby, to clarify its "problematic" (Carr, 1970). Thus, I will show that this problematic arises due to the lack of differentiation between the diverse stratum of the world's constitution, which articulates themselves into genetic and generative investigations that express different levels of analysis of that concept. I will conclude that without making this distinction, which leads to different analysis levels, we will obtain an insufficient comprehension of the concept, such as Husserl developed it throughout different moments of his work.
\end{abstract}

Key Words: World, life-world, horizontality, world's a priori, consistency, progression.

* $\quad$ Autor correspondiente / Corresponding author: cristianmb1984@gmail.com

Este trabajo fue financiado por la Agencia Nacional de Investigación y Desarrollo, Beca de Doctorado Nacional $2018-2022, \mathrm{~N}^{\mathrm{o}} 21181663$. 


\section{INTRODUCCIÓN}

"La pregunta por la esencia del mundo es el problema central de la fenomenología de Edmund Husserl" (Strasser, 1976, p. 152) ${ }^{1}$. De hecho, Husserl describe lo que entiende por mundo de diferentes formas a lo largo de su obra (Strasser, 1976). Por un lado, la palabra mundo es empleada para dar cuenta del mundo de la vida, el cual es en el sentido más pleno del término, un mundo de la cultura (Husserl, 2008a, 288; HUA VI, 191). En este ámbito mundano, puede haber mundos de la vida en el plural en cuanto que cada comunidad humana constituye un mundo relativo a su experiencia intersubjetiva. Por otro lado, no hay una definición unívoca de lo que es el mundo. Husserl lo define como un ser intencional, como el horizonte de los horizontes o como conteniendo una estructura a priori que le otorga una unicidad ante la cual "el plural carece de sentido" (Husserl, 2008a, p. 185; HUA VI, 146). Sin embargo, el mundo también es definido como una adquisición del sujeto y, por consiguiente, es un concepto dinámico que presupone el paso de un premundo (Vorwelt) hacia la constitución del mundo (Welt) en términos más concretos (Walton, 2015; Bower, 2015). Debido a esa variedad de conceptualizaciones acerca del concepto del mundo, se da por un lado una dificultad en la comprensión que se tiene de tal concepto y, por el otro, hay interpretaciones que lo caracterizan incluso como ambiguo. En el presente trabajo introduciré una interpretación comprensiva del concepto de mundo en Husserl. Esta delimitación incluye correlativamente algunas ideas fundamentales tanto de los análisis genéticos como de los generativos de la fenomenología husserliana ${ }^{2}$. Luego, propondré una clave de lectura o interpretación a partir de una diferenciación que se encuentra en Walton (2015) entre los órdenes de la constitución y los de la dación. Mi hipótesis fundamental es que a partir de una consideración que distingue los niveles de la constitución y la dación en torno del concepto de mundo en Husserl, se permite aclarar el sentido fenomenológico de tal concepto y hacer frente a una de las críticas más conocidas en torno a este tema, a saber, el artículo de David Carr "Husserl's Problematic Concept of the Life-World" (1970). Concluiré que la comprensión del concepto fenomenológico de mundo es insuficiente si no se consideran los análisis genéticos y generativos y que, además, la distinción entre los órdenes de la constitución y de la dación pueden clarificar la múltiple estratificación de este concepto en Husserl.

\section{La múltiple estratificación del concepto de mundo en Husserl y su estructura formal}

El concepto de mundo en Husserl ha sido discutido y desarrollado por diferentes fenomenólogos (Heidegger, Merleau-Ponty, Patočka, Carr, Biemel, Moran, Walton). En general, podría decirse que cada uno de los autores posteriores a Husserl profundizó en alguna medida en las diferentes orientaciones, complejidades, "problematicidades" que el concepto fenomenológico de mundo entraña. Para comenzar, es importante señalar que para Husserl el problema del mundo es un problema sobre la subjetividad: "El mundo del que habla Husserl no es nunca la summa rerum o el 'universo' en sentido racionalista. Siempre tiene el sentido de un 'mundo para mí', 'para nosotros, 'para una sociedad de mónadas' y nunca el significado de una totalidad existente de entes. El momento subjetivo es esencial para ese concepto del mundo" (Strasser, 1976, p. 154) ${ }^{3}$. De este modo, para el filósofo alemán el mundo es el "estilo de vida" que se ha configurado a lo largo de la historia del sujeto (Walton, 1993). Como ha indicado Bermes, incluso en el ámbito de una ontología del mundo, tal como es propuesta en Crisis, puede entenderse el concepto de mundo como centrándose en torno al problema de la experiencia:

"En sentido preciso, podría decirse que esta
cosmología es una cosmología radical del ser-
hacia-el-mundo (Zur-Welt-Seins), en la que la
subjetividad no se opone al mundo, sino que el
mundo no puede ser otra cosa que ser-hacia-
el-mundo. Aquí, el mundo no se concibe en
el sentido de una simple totalidad ni como un
espacio universal, sino como una estructura
identificable de la experiencia mundovivida
(der lebensweltlichen Erfahrung)" (Bermes,
2017, p. 103).

De este modo, el problema del mundo es siempre en la fenomenología el problema de la experiencia mundovivida de mundo, es decir, la que se da en la correlación entre la subjetividad y su mundo correlativo.

En cuanto adquisición, el mundo siempre es "suelo familiar de toda experiencia" (Walton, 2015, p. 352, citando Ms A VII 20, 9b: als vertrauten Boden für alle Erfahrung). Cada sujeto, en su vida subjetiva, constituye un mundo que ha venido a ser lo que es debido a una familiaridad que se constituye 
a partir de las habitualidades de la vida subjetiva y los tipos empíricos de los objetos. De ese modo, existe siempre una remisión de la experiencia nueva hacia una experiencia pasada, estableciéndose una coherencia de lo experienciado que se puede atribuir tanto al aspecto noético (la vivencia intencional) como al noemático (el objeto y su remisión a otros objetos). Así, el mundo es un ser constituido de manera dinámica, que encierra una cierta tipología o modo de ser consistente y que deja abierta la posibilidad de siempre llegar a ser más de lo que es en un momento determinado, en el sentido más profundamente teleológico del término. Mundo y conciencia son en Husserl conceptos progresivos.

Junto con lo anterior, porque para el sujeto adulto que reflexiona acerca del mundo, este se presenta siempre como dotado de sentidos prácticos y valóricos, pero también teóricos, la experiencia más inmediata que ese sujeto posee del mundo, en esa etapa del desarrollo de su subjetividad trascendental, lo pone ante un ser que es eminentemente cultural. Este es, según Husserl, el sentido más pleno del mundo (Husserl, 2008a; HUA VI) y ha llegado a ser a partir de la experiencia individual del sujeto en una génesis de sentido que está rodeada de un horizonte de otros sujetos que viven en comunidad y que, al mismo tiempo, constituyen paralelamente el mundo en remisiones hacia ancestros [Vorfahren], quienes han precedido a las subjetividades actuales en la constitución del mundo en cuanto cultura. A este mundo, Husserl le llama mundo de la vida (Husserl, 2008a; HUA VI; HUA IX). Sin embargo, ya que el mundo en el sentido más pleno de la constitución ha llegado a ser, es decir, es un ser teleológicamente constituido, ¿no es posible entonces decir que necesariamente puede hacerse una pregunta retrospectiva acerca del pasado de este mundo en evolución genética de la subjetividad trascendental? Si el mundo es una adquisición, su ser consistente ha venido a ser lo que es gracias a una constitución genética, pero también generacional (o generativa), ¿no son entonces los estratos previos de ese mundo que ha llegado a ser también el tema de la fenomenología?

En la fenomenología de Husserl, el análisis genético es el encargado de develar la estructura temporal de la subjetividad individual a partir de una caracterización del yo como un sustrato de habitualidades. En el polo objetivo, determina al objeto como inserto en un sistema de referencias a partir del cual emerge una típica de su presentación, una tipología empírica que se funda en el sistema de relaciones internas con el mundo circundante que rodea tales objetos y les otorga un estilo determinado (Walton, 1993). En la descripción genética de la subjetividad y el mundo, el "yo" tiene su punto de partida en una autoobjetivación de la subjetividad trascendental ${ }^{4}$, la que se inserta corporalmente en un ámbito de prominencia puramente hylético y afectivo, de pura pasividad asociativa, un estrato de indeterminación al cual Husserl llama premundo [Vorwelt], que se empareja con el concepto de pre-yo [Vor-Ich]. A partir de la correlación de ambos, se constituyen el yo $[I c h]$ y el mundo como tal $[\text { Welt }]^{5}$. El pre-yo es el sujeto corporal que aún no experimenta objetos, pero que de alguna manera posee cinestesias que comienzan a adquirir una coherencia. Este proceso es el origen de la habitualidad. El premundo es el ámbito no-objetivo y aún indeterminado, pero que ya posee una forma de ordenación en cuanto que las cinestesias comienzan ha adquirir consistencia $\mathrm{y}$, de ese modo, a configurar los datos hyléticos en un proceso que terminará con la constitución objetiva (Walton, 2015; Bower, 2015). De este modo, Husserl piensa que el mundo en tanto adquisición, necesariamente tiene un origen trascendental en los estratos primarios de la constitución subjetiva yoica y la del objeto y que, por consiguiente, es necesario caracterizar tales momentos en función de explicar el espesor experiencial y consistente del mundo en su sentido más pleno, es decir, el mundo como mundo de la vida.

A partir de un análisis generativo que se sostiene en la descripción genética de la subjetividad trascendental en su paso del premundo al mundo como tal, Husserl sostiene que el mundo propiamente humano es el así llamado mundo de la vida [Lebenswelt]. El mundo de la vida es constituido por la larga cadena de generaciones humanas que han obtenido el mundo como adquisición intencional tanto en su vida subjetiva individual como colectiva o comunitaria (Held, 1991). Como mencioné anteriormente, este mundo tiene su origen trascendental en el paso del premundo al mundo. Cada estrato del mundo se concatena con otro, en un entramado [Ineinander] que Husserl describirá como el carácter horizóntico del mundo que, además de retrotraer a su génesis, establece el modo de ser [Seinsweise] que manifiesta este mismo mundo en la experiencia. Este carácter horizóntico es una de las características fundamentales de la progresión 
del mundo, pero también caracteriza la experiencia misma de mundo en cuanto tal y la de sus objetos.

El mundo es horizonte en el sentido de que es un entramado [Ineinander] de diferentes campos que van desde la percepción determinada a lo meramente posible en expectación (de lo conocido por experiencias previas) y, desde ahí, hacia lo completamente desconocido. Por ejemplo, sentado aquí en mi escritorio el horizonte más actual se presenta como todo aquello familiar que ha sido constituido por mi experiencia previa y mi corporalidad fungiendo como punto cero de orientación espacial, pero que en primera instancia se manifiesta como aquello que me es más cercano. La mesa sobre la que reposa mi computador, los lápices, mi taza de café, el estuche de mis anteojos, la ventana a partir de la cual miro hacia el horizonte, la playa que está más allá pero que alcanzo con mi vista y que me es familiar porque ya he estado en ella antes, el océano ${ }^{6}$ en su inmensidad que muestra un horizonte lejano y, a partir de ahí, lo desconocido (y, también en dirección contraria, los negocios en la avenida, el cerro y el bosque, luego, lo completamente indeterminado en ese bosque que se extiende a lo largo de una gran cantidad de kilómetros). Así, según Husserl, "avanzando del entorno al entorno del entorno, y así siempre de nuevo, llegamos finalmente al mundo todo" (HUA XXXIX, p. 3627. Citado de Walton, 2012, p. 362). El horizonte como "mundo todo" [die ganze Welt], implica una correlativa experiencia de la totalidad que se da mediatizada a partir de escalamientos de experiencias más remotas del horizonte (Walton, 2012). De ese modo, el mundo contiene un estrato mundovital en el sentido de ser ese mundo constituido en mis experiencias previas como suelo [Boden] y que es dotado de una concordancia [Einstimmigkeit] que le confiere un estilo al mundo que se va complejizando a partir de la determinación y la indeterminación en las que necesariamente se me manifiesta. En cuanto horizonte, el mundo es completamente por determinar en una determinación que nunca acaba. Es decir, el mundo como horizonte es siempre la dinámica que se da entre la vida de conciencia y el mundo como su contrapolo objetivo. Esto da cuenta de una estructura iterativa de la experiencia de horizonte que se muestra en la forma fundamental "y así sucesivamente" [und so weiter] y cuyo correlato experiencial es la posibilidad "se puede siempre de nuevo" [man kann immer wieder] (Walton, 2012). El mundo es un ser teleológico, en el sentido de que siempre -al igual que el objeto- puede ser continuamente enriquecido de maneras diversas y más perfectas, en un escalonamiento de posibilidades de experiencia que se entretejen y sobreponen y que son el fundamento de que el mundo sea en la fenomenología un ser dinámico. Es por eso que el modo de ser del mundo implica que "todo lo que decimos que es en el mundo es más bien encontrado en un apuntar abierto hacia realidades posteriores y revisiones de realidades establecidas" (Knies, 2011, p. 41) ${ }^{8}$.

En conjunto con la estructura dinámica de la horizonticidad, le corresponde al mundo una estructura invariante que Husserl caracteriza en Crisis $\$ 36$ como espacio, tiempo y causalidad. La discusión acerca del modo unitario que le pertenece al mundo se origina en torno a la pregunta por la forma de validación de la experiencia dentro de mi mundo de la vida (lo que Husserl llamará también mundo familiar [Heimwelt]) y, en contraposición con ello, la del mundo ajeno [Fremdwelt] de otras culturas. Según Husserl, pese a la multiplicidad o pluralidad de mundos de la vida, el mundo posee una estructura invariante, que no es el horizonte, y que se mantiene a lo largo de todas las modificaciones que puede sufrir en cuanto tal. Estas estructuras son esenciales en cuanto que constituyen un a priori del mundo que permanece invariante a los cambios (Husserl, 2008a; HUA VI; Walton, 2015). Algo importante que hay que tener en consideración a la hora de pensar estas estructuras formales es que, en primer lugar, son dependientes de mi experiencia cuerpo-vivida en cuanto que mi cuerpo es el centro de la actividad subjetiva en el mundo espacial y temporal y que, por consiguiente, pese a ser la base predada sobre la cual reposa la objetividad espacio-temporal científica, no corresponden a ningún tipo de objetivación o substrucción lógica (Walton, 2015). Lo mismo ocurre con la idea de causalidad. Tiempo y espacio caracterizan la individuación del objeto del mundo $\mathrm{y}$, por tanto, le imprimen su carácter en cuanto tal, en el sentido que la individualidad del objeto depende de su momento temporal y de su lugar en el campo espacial. La causalidad es básicamente la no independencia temporal del objeto que permanece en su identidad a través de los cambios (Walton, 2015) $)^{9}$. Estos elementos formales del mundo se unen para dar origen al concepto de la previsibilidad del mundo: "El mundo tiene una predictibilidad o previsibilidad, que es equivalente a una causalidad de carácter peculiar que escapa a una legalidad 
estricta e incondicionalmente universal porque deja siempre abierta la posibilidad de sucesos causales. No es la causalidad exacta de la naturaleza, sino una 'causalidad morfológica', y, en cuanto surge del proceso de sedimentación de las experiencias en las cuales se nos aparecen conexiones, Husserl señala que es el correlato de la inducción" (Walton, 2015, p. 342). En suma, el componente formal del mundo es el que le otorga un carácter de invariabilidad o unidad [Einheit] que surge a partir de la totalidad enlazada según formas en tanto totalidad unitaria [Alleinheit] (Walton, 2015). De este modo, lo que permanece invariante al mundo en contraposición con los mundos de la vida en plural, es una forma estructural vivencial apriórica cuya determinación depende de una ciencia correlativa trascendental que es la ontología del mundo de la vida (Husserl, 2008a). Como señala Bermes, "Las experiencias mundovividas [Lebensweltliche Erfahrungen] de la temporalidad, la espacialidad, la identidad, la causalidad, o la cercanía y la lejanía, así como de la propiedad y lo ajeno conducen hacia un a priori propio" $(2017,235)$ y, por consiguiente, la tarea de una ontología de ese carácter de mundo, depende específicamente de la determinación fenomenológica de ese a priori.

En el siguiente apartado propondré una clave interpretativa para dar cuenta de los distintos niveles de análisis posibles del mundo y que se relacionan con un orden tanto en la constitución como en la dación.

\section{La clarificación de la estratificación del mundo a partir de la distinción entre los órdenes de la constitución y los de la dación}

El análisis fenomenológico se estructura a partir de distintos niveles de descripción que se correlacionan unos con otros en un entramado que no siempre se vislumbra fácilmente en los textos más conocidos de Husserl, tales como Ideas I, Meditaciones cartesianas o La crisis de las ciencias europeas y la fenomenología trascendental. Esta condición señala una dificultad que radica en que, como expuse en el apartado anterior, el concepto de mundo en Husserl presupone una estratificación múltiple que se caracteriza a partir de un proceso progresivo y escalonado de constitución ${ }^{10}$, así como poseyendo estructuras, tales como el horizonte y un a priori que, respectivamente, subrayan su carácter dinámico y su unidad. Tomando en consideración esta complejidad del concepto de mundo se hace necesaria una clave de interpretación o de lectura que contextualice lo que señala Husserl en cada caso, de modo que se pueda ingresar en un ámbito de comprensión que limite a su mínima expresión la ambigüedad, asumiendo desde el principio que la eliminación de toda ambigüedad es una tarea prácticamente imposible en la interpretación de cualquier filósofo. Esta clave de interpretación puede hallarse en indagaciones filosóficas del fenomenólogo argentino Roberto Walton ${ }^{11}$.

Siguiendo a Husserl, Walton (2012) hace una distinción entre lo que llama los órdenes de la constitución y los de la dación. El primero, corresponde a lo que primariamente se constituye en la experiencia y que puede identificarse con los desarrollos del análisis genético. Genéticamente, lo que primero se constituye es la subjetividad trascendental que se autoobjetiva, insertándose corporalmente en un ámbito de extrañeza, de indeterminación, pero también de prominencias que caracterizan el par pre-yo y premundo. En ese sentido, lo primero que se constituye como tal es el cuerpo vivido. A partir de su objetivación en un ámbito de extrañeza, el cuerpo vivido es el garante de la constitución de los niveles posteriores y más elevados que se funden en el concepto más pleno de mundo de la vida.

Correlativamente, el orden de la dación o de la experiencia corresponde a la unidad de la vida subjetiva trascendental o el sujeto puro. Este sujeto puro es un repositorio y origen de todas las intencionalidades anónimas prerreflexivas o instintivas que han constituido el mundo en torno al cuerpo propio (Walton, 1993). Este sujeto puro que se autoda y, de ese modo, constituye el mundo a partir de la experiencia cuerpovivida, hace que el cuerpo se dé como una unidad noemática trascendental, a partir de la cual el mundo comienza a ser también dado por medio del sentir y el moverse corporales (Walton, 1993). De este modo, el ámbito inicial de la dación es el sujeto puro que constituye un cuerpo para sí y, a partir de ahí, se ordena una serie de daciones que, correlativamente al orden constitutivo, darán el mundo de la vida como el último nivel de la constitución. Sin embargo, esto puede modificarse si tomamos en consideración el análisis generativo del mundo.

El análisis generativo presupone la estructuración de la temporalidad subjetiva de la conciencia, pero lo amplía en el sentido de que incluye la cadena 
de generaciones o de ancestros [Vorfahren] quienes han constituido el mundo previamente en su vida comunitaria. En otras palabras, el análisis generativo es eminentemente histórico y presupone la constitución del mundo cultural, aunque investiga su génesis en la historia de las comunidades humanas (Held, 1991). De este modo, la generatividad refiere un ámbito posterior en el orden de la constitución, en el sentido de que presupone las estructuras genéticas (pero también las estáticas) de la subjetividad y su mundo. Por decirlo con palabras simples, el análisis generativo investiga un ámbito más concreto de la fenomenología, el que determina las estructuras que componen una subjetividad que incluye los elementos más cotidianos de nuestra vida: "el yo trascendental husserliano a la vez dispone de un cuerpo, tiene un estrato intersubjetivo, y no escapa a una historia compartida" (Walton, 1993, p. 16). Como dije anteriormente, el análisis generativo presupone el análisis genético, en el sentido de que el primero investiga un nivel constitutivo superior en relación con el segundo. En ese sentido, es admisible decir que lo último en el orden de la constitución es el mundo de la vida. Sin embargo, si tomamos como modelo la experiencia más concreta del sujeto, entonces veremos que lo dado más inmediatamente en la experiencia, es precisamente este mundo de la vida. Solo la pregunta retrospectiva, que nos pone ante una abstracción, nos permite dar cuenta de que, este mundo familiar directo, el de nuestra experiencia más concreta, ha llegado a ser lo que es en una historia subjetiva de doble dirección: una dirección genética a la que le corresponde un análisis que busca los orígenes desde el premundo al mundo y una dirección generativa que da cuenta de la cadena de generaciones que han constituido el mundo para que llegue a ser lo que es.

La distinción entre el orden de la constitución y el de la dación permite descubrir que el concepto fenomenológico de mundo es progresivo, nivelado de manera múltiple o escalonado, y que presupone la historia trascendental de la subjetividad en términos individuales y colectivos. Cada estrato del mundo, sea de los niveles genéticos previos, tales como el premundo indiferenciado y el paso al mundo del niño, del adolescente y del adulto, hasta la constitución de un mundo intersubjetivo que se caracteriza por ser común a una comunidad de sujetos y que Husserl llama mundo de la vida (cultural) puede ser entendido a partir de esta diferenciación en tanto que aclara el nivel del análisis fenomenológico y, por consiguiente, circunscribe analítica y abstractivamente diferentes estratos de la investigación ${ }^{12}$. $\mathrm{Si}$ a esta estructura le agregamos la determinación del modo de ser del mundo, en cuanto horizonte y en cuanto estructura apriórica, podemos entonces llegar a una caracterización sistemática del mundo que permite clarificar y despejar objeciones en torno al concepto de mundo en Husserl, tales como la de David Carr (1970) que lo caracteriza como problemático. En la siguiente sección mostraré cómo a partir del reconocimiento de los distintos niveles de estratificación del mundo, en cuanto al orden constitutivo y de dación, puede despejarse la objeción de que el mundo es un concepto problemático en la fenomenología de Husserl.

\section{El aparentemente problemático concepto de mundo de la vida en Husserl}

David Carr (1970) en su artículo "Husserl's Problematic Concept of the Life-World", critica a Husserl por las "muchas fallas y confusiones de su exposición" (Carr, 1970, p. 331) ${ }^{13}$ del concepto de mundo de la vida en su obra Crisis de las ciencias europeas y la fenomenología trascendental. Los principales errores que Carr atribuye a Husserl en la exposición del concepto de mundo de la vida son: a) la falta de articulación e incompatibilidad de los subconceptos que subyacen al concepto más general de mundo de la vida, tales como mundo de la experiencia directa y mundo de la cultura; b) la imposibilidad del mundo cultural de ser histórica y sociológicamente no relativo, dado que le pertenece un ámbito de lo intuitivamente dado que no cambia con el tiempo ni las circunstancias (Carr 1970). Ampliando un poco la interpretación, lo que para Carr parece problemático es que, por un lado, Husserl en Crisis apela a que el mundo de la vida es el ámbito siempre más familiar e intuitivamente dado de manera inmediata (siendo este mundo al mismo tiempo un mundo cultural) y que, por el otro, plantea que este mundo funciona como siendo predado [vorgegeben] para la ciencia, en tanto actividad teorética que se basa en este mismo mundo, como su ámbito de fundamentación primaria, como suelo [Boden] y que, por consiguiente, permanece invariante en sus posibles modificaciones o nuevos sentidos.

Los argumentos de Carr para esgrimir su interpretación es que existe una fractura entre, por un lado, el entendimiento de ese mundo primario de experiencia inmediata intuitiva, del "universo de lo 
que es intuible en principio" (Carr, 1970, p. 334) ${ }^{14}$, el que se identifica con el mundo de la percepción, entendido como lo que me es accesible de manera más cercana y familiar, y que posee una estructura inmodificable de lo subjetivo-relativo, y, por otro lado, el mundo cultural que incluye instituciones, organizaciones políticas, literatura, religión, que constituyen "la cosa más lejana a una fenomenología de la percepción" (Carr, 1970, p. 337) ${ }^{15}$. El origen de la problemática fundamental de la descripción del mundo de la vida radica en que, pese a que el mundo de la vida es dependiente del mundo de la percepción, el mundo cultural es idéntico con ese mundo intuitivo, aunque poseen formas distintas de dación. Por ejemplo, mientras que la percepción inmediata del pollito de mar (phalaropus tricolor) corriendo rápidamente por la orilla de la playa se da a partir se matizaciones perceptivas y de síntesis en la multiplicidad de esas matizaciones, con un horizonte interno (sus múltiples apariciones en relación con la unidad del objeto) y externo (un contexto de otros objetos que son tributarios de la experiencia del objeto), una institución gubernamental de la zona donde habito se me da en la forma de una institución que es mediatizada por un contexto generativo de constitución y de estructuras culturales nacionales. En el primer caso, la experiencia es inmediata, mientras que en el otro, la experiencia es mediatizada por la tradición, la comunidad y el lenguaje. Este problema se hace complejo cuando Carr subraya que para Husserl el mundo cultural es a su vez presupuesto por el mundo científico.

El mundo científico es una "substrucción lógica" 16 [logische Substruktion] sobre la subjetivo-relatividad del mundo de la vida. De tal modo, se da un contraste entre el mundo de la vida siempre accesible de manera intuitiva y el mundo científico que es mediatizado por la objetivación que la ciencia hace sobre la subjetivo-relatividad del mundo de la vida (Husserl, 2008; HUA VI; Biemel, 1958). Si tomamos en consideración que el mundo de la vida es para Husserl la suma de ese mundo de experiencia inmediata perceptiva y el mundo cultural, y que, por este mismo motivo, ambas caracterizaciones del mundo le sirven como base, es decir, son predadas para la experiencia científica teórica, entonces la ciencia corresponde a un estrato ulterior en la constitución del mundo. Según Carr, el problema radica específicamente en que, si la ciencia es una actividad teórica que se enmarca dentro de la cultura -al menos, del mundo de la vida occidental-, entonces habría que preguntarse cómo se tiene que entender la predación del mundo cultural respecto del mundo de la ciencia que, en cierto sentido, es englobado por este mundo que, a su vez, se posa sobre el terreno del mundo de la percepción (Carr, 1970). En otras palabras, tomando en consideración que el mundo de la ciencia es una substrucción que se realiza en el campo del resultado constitutivo que unifica los mundos de la percepción y de la cultura, entonces la ciencia corresponde a un estrato terciario y dependiente en su realización como actividad intencional de la constitución de esos mundos como dados de antemano.

Una respuesta posible a esta problemática, al menos en torno a la diferenciación de conceptos utilizados por Husserl para caracterizar el mundo, es que en el análisis fenomenológico cada concepto está relacionado con un momento diferente de la constitución y de la dación. Ya que el mundo es un ser en progresivo desarrollo genético y generativo, cada vez que Husserl se refiere al mundo, este no puede ser encasillado completamente en ninguno de los niveles descriptivos que lo caracterizan. De este modo, cuando Husserl señala la experiencia intuitiva directa y la correlaciona con el mundo cultural, de lo que se trata es que el mundo de la percepción en cuanto abstracción, es un componente necesario para la determinación del objeto cultural. En otras palabras, sin una aprehensión intuitiva primaria del objeto en una red horizóntica de experiencias previas y posteriores, no puede haber objeto cultural como tal. Asimismo, ese objeto siempre llega a ser en una historia generativa que incluye la cadena de generaciones que constituye el mundo de una manera comunitaria determinada y que corresponde a la multiplicidad del mundo entendido en términos de distintas culturas (históricas y territoriales) ${ }^{17}$. El mundo de la ciencia, en tanto substrucción lógica sobre el mundo de la vida y que corresponde a la pregunta por la objetividad del mundo uno ${ }^{18}$, es un nivel ulterior de la constitución del mundo que, a juicio de Husserl, tiene el problema de escapar al ámbito intuitivo, como se señala en Crisis §34d. En este sentido, el mundo de la ciencia, pese a tener su suelo en el mundo de la vida que le es predado, hace necesariamente una anulación de este mundo al tratar de sobreponer su metódica investigativa sobre la subjetivo-relatividad. Por consiguiente, pese a que el mundo de la cultura incluya como posibilidad teórica el desarrollo de una ciencia objetiva, el mundo objetivo que la ciencia intenta construir 
por debajo del mundo de la vida y, de esta manera, desplazarlo a un segundo plano, pertenecería a un polo ideal abstractivo que jamás experimentamos en términos concretos. En otras palabras, es un mundo mediatizado y producido por la metodología científica objetiva.

La diferencia fundamental entre los distintos estratos constitutivos del mundo, incluso el de la posibilidad de un mundo científico objetivo, radica en el modo en que uno de esos estratos se instaura sobre el suelo del otro, en un orden teleológico universal infinito y que, por consiguiente, las diferenciaciones que se establecen entre un mundo y otro, son de carácter interpretativo, en el sentido de que pertenecen a esos mundos como caracterizaciones abstractas que permiten delimitar uno de sus estratos, el que será objeto de uno de los niveles de la descripción fenomenológica. Además, en términos constitutivos, puede establecerse una cadena que va desde el premundo, al mundo de la experiencia sensible inmediata y, de este, al del mundo de la cultura comunitaria. La idea de la ciencia y el correlativo establecimiento de un mundo objetivo en cuanto pregunta fundamental, es una de las posibilidades que surgen en el seno de un mundo cultural específico, en este caso, la Antigua Grecia ${ }^{19}$. Sin embargo, y pese a que nuestro mundo occidental es un mundo mediatizado por la idea de la ciencia, en tanto posibilidad, ella no es necesaria para que exista un mundo propiamente tal, pues todo ser humano, en tanto ser cultural de una historia generativa, posee mundo: "Es esencialmente imposible encontrar hombres en cualquier estado 'pre-mundano', porque ser humano, ser consciente de sí mismo como hombre y existir como un yo humano, es precisamente vivir sobre la base de un mundo" (Landgrebe, 1940, p. 53). Por consiguiente, pese a la importancia que aquí solo puede ser indicada, la idea de la ciencia y su correlativo mundo objetivo constituyen efectivamente un nivel ulterior de la constitución del mundo pero que, necesariamente, es justificado en el campo apriórico del mundo, en cuanto que el espacio-tiempo y la causalidad se constituyen en primer lugar a partir de mi experiencia cuerpovivida $\mathrm{y}$, por consiguiente, toda substrucción lógica acerca de tales estructuras siempre posee el carácter de lo mediado, es decir, de lo no intuitivo $\mathrm{y}$, por consiguiente, fundado sobre un mundo que es primariamente intuitivo, aquel que he caracterizado en este texto como mundo, pero que en el nivel más pleno, es llamado por Husserl mundo de la vida y que posee una ciencia que se encarga de ella que es la fenomenología trascendental en tanto que ontología del mundo de la vida.

\section{CONCLUSIÓN}

El concepto de mundo en Husserl es problemático en cuanto presupone prácticamente toda la vida y evolución filosófica del pensamiento del filósofo alemán. Todo intento de deslindar y, a partir de ello, criticar esta noción, ya sea como confusa (mundo de la vida) o relativista (mundos de la vida en plural), etc., se sustenta sobre una mirada insuficiente de la amplitud y la dificultad que este concepto posee en el trabajo husserliano. Como señalé, es posible aproximarse al concepto del mundo en Husserl tomando en consideración la ordenación correspondiente a la idea de constitución, así como respecto del orden de la dación. El mundo es constituido y dado a partir de múltiples niveles en los dos sentidos. Además, el mundo es siempre un horizonte dinámico que pone en juego lo determinado e indeterminado, lo conocido y lo desconocido. Pese a que el mundo puede ser pensado en el plural como mundos de la vida de las comunidades intersubjetivas históricas y actuales, le corresponde a cada mundo de la vida una estructura formal que sobrepasa la relatividad a la que estos están sometidos en cuanto plurales. Esas estructuras son estructuras vividas, dependientes de la vida trascendental corporal de la subjetividad y son el fundamento de la substrucción lógica posterior que realiza la ciencia sobre el mundo de la vida.

En sí mismo, el problema de la relación entre el mundo de la vida y el mundo de la ciencia fueron tratados por Husserl, principalmente, en su obra Crisis. No obstante, el problema del mundo no está completamente resuelto, ni expuesto en sus detalles, en dicha obra. Tal como ha señalado Bermes, el problema del mundo en Husserl se halla más bien en sus escritos inéditos (2017). Como vimos, pese a la problemática que se presenta en relación con, por un lado, el mundo perceptivo y cultural y, por el otro, el mundo científico, el problema del mundo en fenomenología sobrepasa tal problemática. Esa consideración ha sido la que se ha expuesto en este trabajo, a saber, la de un concepto de mundo que supera en términos de contenido los mundos específicos, los mundos relativos y se orienta en una comprensión fenomenológica que hace del mundo 
su tema de investigación. Este es el problema de la ontología del mundo de la vida, con su a priori y su estructura horizóntica.

Para finalizar, solo me resta decir que el problema del mundo en Husserl está siempre correlacionado con el problema de la subjetividad y, más aún, la correlación entre subjetividad y mundo constituye el leitmotiv de su filosofía: "La primera vez que surgió este a priori de la correlación entre objeto de experiencia y modos de darse (durante la elaboración de mis Investigaciones lógicas alrededor del año 1898) me conmovió tan profundamente [errchütterte mich so tief] que desde entonces todo el trabajo de mi vida fue dominado por la tarea de una elaboración sistemática de este a priori de correlación" (Husserl, 2008, p. 207; HUA VI, p. 169). En otras palabras, la determinación de la subjetividad y su mundo correlativo, en su elaboración sistemática, no es el resultado de una simple deliberación en Investigaciones lógicas, Ideas I o la Crisis, sino que un trabajo que se extendió a lo largo de toda su vida y obra filosóficas.

\section{TEXTOS DE HUSSERL}

HUA IV Ideen zur einer reinen Phänomenologie und phänomenologischen Philosophie. Zweites Buch: Phänomenologische Untersuchungen zur Konstitution. Editado por Marly Biemel. The Hague, Netherlands: Martinus Nijhoff, 1952. [ISBN: 978-90-247-0218-3]

HUA VI Die Krisis der europäischen Wissenschaften und die transzendentale Phänomenologie. Editado por Walter Biemel. The Hague, Netherlands: Martinus Nijhoff, 1976. [ISBN: 978-90-247-0221-3]

HUA XV Zur Phänomenologie der Intersubjektivität. Texte aus dem Nachlass. Dritter Teil. 192935. Editado por Iso Kern. The Hague, Netherlands: Martinus Nijhoff, 1973. [ISBN: 978-90-247-5030-6]

HUA XXXIX Die Lebenswelt. Auslegungen der vorgegebenen Welt und ihrer Konstitution. Texte aus dem Nachlass (1916-1937). Editado por Rochus Sowa. New York: Springer, 2008. [ISBN: 978-1-4020-6476-0] 


\section{REFERENCIAS}

Bermes, C. (2017). Die Krisis der europäischen Wissenschaften und die transzendentale Phänomenologie. En Husserl Handbuch (pp. 90-103). J.B. Metzler.

Bermes, C. (2017b). Die Lebenswelt. En Husserl Handbuch (pp. 230-236). J.B. Metzler.

Biemel, W. (1959). Die entscheidenden Phasen der Entfaltung von Husserls Philosophie. Zeitschrift für philosophische Forschung, 13 (2), 187-213.

Bower, M. (2015). Husserl's Concept of the Vorwelt and the Possible Annihilation of the World. Research in phenomenology, 45, 108-126, https://doi.org/10.1163/15691640-12341304

Carr, D. (1970). Husserl's Problematic Concept of the Life-World. American Philosophical Quarterly, 7 (4), 331-339, http://www. jstor.org/stable/20009365

Guiñez, P. (2017). Paradoja de la subjetividad y apercepciónmundanizante. Una incursión en la humanidad de lasubjetividad trascendental [Libro electrónico]. En A. Gatica (Ed.), Incursiones fenomenológicas sobre el análisis intencional,la reducción y la angustia ( $1^{\mathrm{a}}$ ed., pp. 61-82). Teseo Press, https://www.teseopress. com/incursiones/front-matter/19-2/

Held, K. (1991). Heimwelt, Fremdwelt, die eine Welt. Phänomenologische Forschungen, 24/25, 305-337, https://www. jstor.org/stable/24360333

Husserl, E. (1940). Grundlegende Untersuchungen zum Phänomenologischen Ursprung der Räumlichkeit der Natur. En M. Farber (Ed.), Philosophical Essays in Memory of Edmund Husserl ( $1^{\mathrm{a}}$ ed., Vol. 1, pp. 307-325). Harvard University Press.
Husserl, E. (2008). La crisis de las ciencias europeas y la fenomenología trascendental ( $1^{\mathrm{a}} \mathrm{ed}$.). Prometeo Libros.

Knies, K. (2011). Three Views of the One True World and What They Make of Mere Worldviews: A Husserlian Approach to Weltanschauung. Humana.Mente Journal of Philosophical Studies, 18, 39-54.

Landgrebe, L. (1940). The World as a Phenomenological Problem. Philosophy and Phenomenological Research, 1 (1), 38-58, https://www.jstor.org/stable/2103195

Lee, N. (2020). The Pluralistic Concept of the Life-World and the Various Fields of the Phenomenology of the Life-World in Husserl. Husserl Studies, 36, 47-68.

Strasser, S. (1976). Der Begriff der Welt in der phänomenologischen Philosophie. Phänomenologische Forschungen, 3, 151-179.

Szilazi, W. (1959). Einführung in die Phänomenologie Edmund Husserls. Max Niemeyer Verlag.

Walton, R. (1993). Husserl, mundo, conciencia y temporalidad ( $1^{\mathrm{a}}$ ed.). Editorial Almagesto.

Walton, R. (2004-2005). Egología y generatividad. Seminarios de filosofía, 17-18, 257-286, https://repositorio.uc.cl/xmlui/ bitstream/handle/11534/9982/000398672.pdf

Walton, R. (2015). Intencionalidad y horizonticidad ( $1^{\mathrm{a}} \mathrm{ed}$.). Editorial Aula de Humanidades.

Zahavi, D. (2003). Husserl's Phenomenology. USA: Stanford University Press. 


\section{NOTAS}

$1 \quad$ Die Frage nach dem Wesen der Welt is das Zentralproblem der Phänomenologie Edmund Husserls.

2 Para el problema sobre los análisis genéticos y generativos en la metodología husserliana, ver Steinbock, 1995; Walton,

2015.

3 Die Welt, von der Husserl spricht, ist niemals die "summa rerum" oder das "Universum" im rationalistischen Sinn. Sie hat immer den Sinn einer "Welt für mich", "für uns", "für eine Gemeinschaft von Monaden" und niemals die Bedeutung einer an sich bestehenden Allheit von Seienden. Das subjektive Moment ist für diesen Begriff der Welt wesentlich.

$4 \quad$ Ver, Walton, 2004; Guiñez, 2017.

5 Acerca del tema de la vida subjetiva en sus estratos instintivos, ver Walton, 2004; Rabanaque, 2003. Respecto del concepto de premundo, ver Bower, 2015.

6 Es posible pensar que el océano, tal como el cielo, constituye un límite respecto de la tierra en tanto que proto-arca [UrArché] (Husserl, 1940, 308). Ya que la experiencia cuerpo-vivida mediante las cinestesias solo nos permite habitar la tierra en nuestra experiencia inmediata porque el cielo es una dimensión límite en tanto que imposible de habitar, es decir, el cielo solo es posible de experimentar a partir de la imaginación (no tenemos alas como las aves, pero podemos imaginarnos dotados de ellas para volar en la dimensión celeste), en analogía con el cielo, el océano también es un ámbito límite, pues se presenta primero como una superficie cuya profundidad es inexplorada y, en la cual, solo podemos explorar a partir de la utilización de trajes o naves de inmersión que nos impide el contacto natural directo con su espesura (tal como los animales y plantas marinas experimentan en forma directa). Sin embargo, esto es solo una indicación y todo desarrollo debe ser llevado a cabo a partir de reflexiones posteriores. $7 \quad$ Von Umgebung zu Umgebung der Umgebung fortschreitend, und so immer wieder, kommen wir doch schließlich auf die ganze Welt.

$8 \quad$ Everything we say to be in the world is rather encountered in an openness pointing to further realities and revisions of established realities.

9 La forma causal también es el sustrato de la sustancialidad. Sin embargo, dejaré el desarrollo de este concepto para otro momento.

10 Constitución es el nombre para un concepto técnico en la fenomenología y debe entenderse como "un proceso que permite la manifestación y la significación [...] un proceso que permite que lo que es constituido aparezca, se despliegue, se articule y se muestre a sí mismo como es" (Zahavi, 2003, 73) a process that allow for manifestation and signification [...] a process that permits that which is constituted to appear, unfold, articulate, and show itself as what it is. En términos fundamentales, incluye tanto al sujeto como al mundo: "El término 'constitución' siempre significa ambos: la constitución de la unidad del ego y experiencia constitutiva del mundo unificado" (Szilazi, 1959, 93) Unter der Bezeichnung "Konstitution” ist ständig beides zu verstehen: Konstitution der Einheit des Ichs und konstitutive Erfahrung der einheitlichen Welt.

11 Lee ha señalado una idea similar a la que persigo acá. El autor propone que el concepto de mundo de la vida debe entenderse como siendo esencialmente múltiple y, por consiguiente, sostiene que existen diversos campos de la fenomenología que se dedican a estudiar el mundo de la vida. Este trabajo, sin explorar el problema bajo la misma consideración, está en sintonía con lo propuesto recientemente por ese autor. Ver Lee 2020.

12 Esta es precisamente la tesis de Lee, cuando señala que una comprensión suficiente del concepto de mundo en Husserl debe considerar sus múltiples significados (Lee, 2020).

13 "Many faults and confusions in his exposition".

14 "Universe of what is intuitable in principle".

15 "The farthest thing from a phenomenology of perception".

16 En la traducción de Crisis de Iribarne se traduce Substruktion por sustracción, dando a entender que lo que la ciencia hace es quitarle un elemento al mundo de la vida o, simplemente, despojándolo de algo -su subjetivo-relatividad-. Considero que este uso terminológico es incorrecto. Pienso que el concepto puede traducirse sin más por substrucción para traducir el alemán, el cual tiene el significado de basamento [Unterbau], fundación [Grundbau]. David Carr traduce el alemán Substruktion por substruction, término que significa construir por debajo como base o fundamento. En español, el concepto substrucción se emplea como base a partir de la cual algo se construye (Fuentes: https://www.duden.de/rechtschreibung/Substruktion ; https://www.collinsdictionary. $\mathrm{com} / \mathrm{es} /$ diccionario/ingles/substruct ; file:///Users/crismartinezb/Downloads/las-bvedas-de-substruccin-del-circo-romano-de-tarragona-0.pdf Consultado el 3 de diciembre de 2020).

17 Ver Husserl, 1973 (HUA XV); 2008b (HUA XXXIX).

18 Es importante subrayar que, para Husserl, la tarea de una ciencia del mundo es una tarea fenomenológica. Y, esto, porque la fenomenología es la que se encargaría de determinar y esclarecer el carácter formal apriórico del mundo, con sus estructuras espacio-temporales y la causalidad, que son la base mundovivida de la objetivación científica sobre esas mismas estructuras. Es posible destacar que, como señalé en la sección anterior, la unicidad del mundo es pensada fenomenológicamente a partir de ese a priori. Su carácter dinámico, a partir de la estructura de la horizonticidad. Ver Husserl, 2008a (HUA VI); 2008b (HUA XXXIX); Walton, 2015.

19 Ver el apéndice III de HUA VI. Hay traducción al español de Rosemary Rizo-Patrón en un tomo compilatorio llamado Textos Breves (1887-1936). 